\title{
Current therapeutic options for coronavirus disease 2019 (COVID-19) - lessons learned from severe acute respiratory syndrome (SARS) and Middle East Respiratory Syndrome (MERS) therapy: a systematic review protocol
}

\author{
Zai-Li Zhang ${ }^{1 \#}$, Han Zhong ${ }^{1 "}$, Yang-Xi Liu ${ }^{1}$, Ke-Jia Le ${ }^{1}$, Min Cui ${ }^{1}$, Yue-Tian Yu ${ }^{2}$, Zhi-Chun Gu ${ }^{1}$, \\ Yuan $\mathrm{Gao}^{2}$, Hou-Wen Lin ${ }^{1}$ \\ ${ }^{1}$ Department of Pharmacy, ${ }^{2}$ Department of Critical Care Medicine, Renji Hospital, School of Medicine, Shanghai Jiao Tong University, Shanghai, \\ China \\ Contributions: (I) Conception and design: ZC Gu; (II) Administrative support: YT Yu, Y Gao, HW Lin; (III) Provision of study materials or patients: \\ None; (IV) Collection and assembly of data: H Zhong, ZL Zhang; (V) Data analysis and interpretation: ZC Gu, ZL Zhang; (VI) Manuscript writing: \\ All authors; (VII) Final approval of manuscript: All authors. \\ "These authors contributed equally to this work. \\ Correspondence to: Zhi-Chun Gu, MD. Department of Pharmacy, Renji Hospital, School of Medicine, Shanghai Jiao Tong University, Shanghai \\ 200127, China. Email: guzhichun213@163.com; Yue-Tian Yu, MD, PhD. Department of Critical Care Medicine, Renji Hospital, School of \\ Medicine, Shanghai Jiao Tong University, Shanghai 200127, China. Email: fishyyt@sina.com.
}

Background: Coronavirus disease 2019 (COVID-19), also known as severe acute respiratory syndrome coronavirus 2 (SARS-CoV-2) infection, first manifested in December 2019, and spread rapidly worldwide. Facing this lethal disease, there is an urgent need to develop potent therapies against SARS-CoV-2 infection. SARS-CoV-2 phylogenetically and symptomatically resembles SARS-CoV and Middle East Respiratory Syndrome Coronavirus (MERS-CoV). Numerous agents have been utilised during the severe acute respiratory syndrome (SARS) and Middle East Respiratory Syndrome (MERS) epidemics, which may show some benefit against SARS-CoV-2.

Methods: MEDLINE, EMBASE, Cochrane Library, CBM Disc, China National Knowledge Infrastructure, Wanfang Data, and the China Science and Technology Journal Database will be searched. Manual searches will be conducted by searching pre-printing websites, clinical trial registers, and screening the reference lists of inclusive studies. The screening of all citations and the selection of inclusive articles will be conducted by two reviewers. Randomised controlled trials (RCTs) and controlled cohort studies reporting antiviral therapies, including ribavirin, remdesivir, lopinavir/ritonavir, arbidol, chloroquine, hydroxychloroquine, and interferon, for SARS, MERS, and COVID-19 will be included. The primary outcomes will be mortality, incidence of acute respiratory distress syndrome, and utilisation of mechanical ventilation and intensive care unit admission. The secondary outcomes will be improvement in symptoms and chest radiography results, virus clearance, changes in blood test results, and serum tests. The quality of the retrieved RCTs and observational studies will be appraised according to the Cochrane risk of bias tool and the Newcastle-Ottawa Scale, respectively. If feasible, we will perform a fixed- or random-effects metaanalysis.

Discussion: This systematic review and meta-analysis will summarise all the available evidence for the efficacy and safety of current therapeutic options in SARS-CoV, MERS-CoV, or SARS-CoV-2-infected patients. The findings of this study may inform subsequent antiviral interventions for patients with COVID-19.

Study registration: The protocol of this study has been submitted to the PROSPERO platform (https://www.crd.york.ac.uk/PROSPERO/), and the registration number is CRD42020168639. 
Keywords: Coronavirus disease 2019 (COVID-19); severe acute respiratory syndrome (SARS); Middle East Respiratory Syndrome Coronavirus (MERS); therapeutic options; efficacy; safety

Submitted Mar 09, 2020. Accepted for publication Oct 18, 2020.

doi: $10.21037 /$ atm-20-2340

View this article at: http://dx.doi.org/10.21037/atm-20-2340

\section{Introduction}

The coronavirus disease 2019 (COVID-19) is an emerging infection caused by severe acute respiratory syndrome coronavirus 2 (SARS-CoV-2), a novel lineage B betacoronavirus $(1,2)$. As of $5 \mathrm{July}, 2020$, a total of 11,125,245 confirmed COVID-19 cases had been reported, with 528,204 fatal cases (3). SARS-CoV-2 possesses a genome structure similar to that of SARS-CoV and Middle East Respiratory Syndrome Coronavirus (MERS$\mathrm{CoV}$ ) (4). Meanwhile, the clinical features of patients with COVID-19 are very similar to those in SARS and MERS (5). Common symptoms include fever, fatigue, dry cough, dyspnoea, and anorexia (6). Most patients have mild and reversible disease. However, 14\% of cases are severe, with pneumonia and shortness of breath, while approximately $5 \%$ of patients have critical disease, including acute respiratory distress syndrome (ARDS), septic shock, and multiple organ dysfunction syndrome (7).

Given the fast transmission of this potentially lethal disease and the lack of approved antiviral treatment for COVID-19, it is crucial to deploy effective therapies as a high priority (8). As COVID-19 phylogenetically and symptomatically resembles SARS and MERS, a variety of agents have been tried based on clinical experience from SARS and MERS, including broad-spectrum antiviral agents (ribavirin) (9), innate immune responders to virus infection (interferons) $(10,11)$, HIV protease inhibitors (lopinavir and ritonavir) $(12,13)$, and combinations of the above therapeutics $(14,15)$. In addition, antiviral treatments, including spike $(\mathrm{S})$ protein angiotensin-converting enzyme 2 (ACE2) blockers (e.g., chloroquine) $(16,17)$, nucleoside analogue (remdesivir) (18), and viral fusion blockers (arbidol) (19), have been reported to exhibit antiviral activity against SARS-CoV-2 in vitro and in vivo (20). Herein, these treatments have been chosen as possible treatment options for COVID-19 (21).

Few clinical trials evaluating therapeutic agents for COVID-19 have been reported, demonstrating inconclusive clinical outcomes $(14,22)$. Therefore, we plan to conduct this review to summarise the effectiveness and safety of current treatments for SARS and MERS in addition to COVID-19, in an attempt to identify promising therapies for SARS-CoV-2-infected patients.

We present the following article in accordance with the PRISMA-P reporting checklist (available at http://dx.doi. org/10.21037/atm-20-2340).

\section{Methods}

\section{Search strategy}

This study will be conducted according to the Preferred Reporting Items for Systematic Reviews and MetaAnalyses (PRISMA) (23), and a previously registered protocol (PROSPERO: CRD42020168639). We will search MEDLINE via PubMed, EMBASE, Cochrane Library, CBM disc via SinoMed, China National Knowledge Infrastructure, Wanfang Data, and the China Science and Technology Journal Database. The search strategy will include patient-related terms (COVID-19, severe acute respiratory syndrome coronavirus 2 , severe acute respiratory syndrome, Middle East Respiratory Syndrome Coronavirus, coronavirus) and intervention-related terms (interferon, HIV protease inhibitors, lopinavir, ritonavir, ribavirin, remdesivir, saquinavir, darunavir, atazanavir, indinavir, fosamprenavir, nelfinavir, bictegravir, dolutegravir, arbidol, beclabuvir, and chloroquine), using both Medical Subject Headings (MeSH) and free-text terms. The detailed search strategy is available in Table $\mathrm{S} 1$.

Complementary searches will be also conducted by manually searching the content of medical journals (e.g., New England Fournal of Medicine, The Fournal of American Medical Association, Lancet, Chinese Fournal of Infectious Disease, and the Chinese fournal of Tuberculosis and Respiratory Diseases) published after December 2019, as well as the news of the World Health Organization (WHO) and the Chinese Centre for Disease Control and Prevention (CDC). For the purpose of identifying all potentially relevant articles, we will also screen all the reference lists of the retrieved articles. The websites of MedRxiv (https://www. medrxiv.org), SSRN (https://www.ssrn.com/index.cfm/en), 
ChemRxiv (https://chemrxiv.org), BioRxiv (https://www. biorxiv.org), and ChinaXiv (http://www.chinaxiv.org/home. $\mathrm{htm}$ ) will be searched for articles in preprint, while the websites http://clinicaltrials.gov/, http://www.isrctn.com/, and http://www.chictr.org.cn/ will be searched for ongoing trials.

\section{Selection of studies}

Two reviewers (ZL Zhang and H Zhong) will independently assess the titles and abstracts to determine their eligibility for inclusion. Subsequently, full papers will be retrieved and assessed according to the inclusion and exclusion criteria. We will include studies that (I) are conducted among adult patients with SARS, MERS, or COVID-19; (II) assess the effectiveness and safety of anti-coronavirus agents versus placebo or standard treatment regimens; and (III) have a randomised control or cohort design. We will exclude studies that (I) are in-vitro or in-vivo studies; (II) are reviews, guidelines, comments, case series, or case reports; (III) lack a control group; or (IV) lack quantitative or qualitative outcomes of interest. Any disagreements will be resolved by a corresponding author $(\mathrm{ZC} \mathrm{Gu})$.

\section{Study outcomes}

The primary outcomes will be (I) coronavirus-related mortality, (II) incidence of ARDS, (III) utilisation of mechanical ventilation, and (IV) intensive care unit (ICU) admission. The secondary outcomes will be (I) time to become afebrile, (II) drop of viral load, (III) improvement in chest radiography results, (IV) number of patients with anaemia, (V) renal dysfunction rate, (VI) changes in liver enzymes, blood count, and electrolyte levels, and (VII) the increase in creatine kinase.

\section{Data extraction}

We will extract data using a well-designed form, including study characteristics (investigator, publication year, study location, study design, sample size), population characteristics (sex, age, diagnosis, comorbidities), intervention characteristics (therapeutic agents, administration start date, dosage, treatment duration, concomitant medication), clinical outcomes (mortality, incidence of ARDS, mechanical ventilation usage, ICU admission, hospital stay, reduced viral load, clinical improvement, improvement of symptoms, and time to become afebrile).

\section{Quality assessment}

The methodological quality of randomised clinical trials (RCTs) will be assessed using the Cochrane Collaboration Risk of Bias Tool (24). The following items will be appraised: adequacy of randomisation, concealment of allocation, blinding of outcome assessors, completeness of data, and selective outcome reporting (25). The risk of bias of retrospective studies will be evaluated according to the Newcastle-Ottawa Scale (NOS) (26). The items with regard to patient selection, comparability between groups, and outcome or exposure factor assessment will be estimated (27). The risk of bias of individual studies will be rated as low (NOS scores $\geq 7$ ), moderate ( $4 \leq$ NOS scores $\leq 6$ ), or high (NOS scores $\leq 3$ ). For each study, the quality characteristics will be rated as low risk of bias, moderate risk of bias, high risk of bias, or unclear.

\section{Statistical analysis}

The data will be analysed according to the recommendations of the Cochrane Handbook for Systematic Reviews of Interventions (28), and with the use of STATA software (version13, Statacorp, College Station, Texas, USA). Heterogeneity will be assessed using the Cochrane $\mathrm{I}^{2}$ statistic, with a value of $>50 \%$ indicating statistical heterogeneity (29). Dichotomous data will be expressed as relative risk (RR) with $95 \%$ confidence interval (CI). Continuous variables will be calculated as weight mean difference (WMD) with 95\% CIs. A fixed-effect or randomeffects model will be used to analyse statistics. The randomeffects model is the preferred choice while heterogeneity among studies is significant (28). Subsequently, we will perform subgroup analyses according to indications (COVID-19, SARS, MERS), severity of infection (severe, moderate, or mild), treatments (interferon, lopinavir, ritonavir, ribavirin, remdesivir, arbidol, chloroquine), dosage (high or standard), administration route (inhale, infusion, or oral), and initial date of administration (within $48 \mathrm{~h}$ or delayed). We also plan to conduct sensitivity analyses by omitting individual studies in order to assess the confidence of the results. Sensitivity analyses will be conducted by excluding studies with a high risk of bias or small sample size. Publication bias will be evaluated by visual funnel plots, as well as quantitative Begg's and Egger's tests (23). A P value $<0.05$ will indicate a statistically significant difference. 


\section{Discussion}

COVID-19 has led to a global pandemic (3), and numerous antiviral agents have been considered for the treatment against the novel causative virus, SARS-CoV-2. Among the treatment possibilities based on experience with previous coronaviruses, remdesivir $(30,31)$, chloroquine, hydroxychloroquine $(16,17)$, lopinavir/ritonavir $(12,13)$, ribavirin (9), arbidol (19), and interferons $(10,11)$ have been tested most extensively. Several systematic reviews and meta-analyses have assessed the efficacy and safety of antiviral agents in patients with SARS, MERS, or COVID-19. However, the limitations of these studies should not be neglected.

Momattin et al. conducted a systematic review to summarise potential treatments for MERS based on earlier studies of SARS (22). They determined that ribavirin may improve SARS-CoV infection in $71.4-80 \%$ of patients, and reduce ICU admission rates to $13-20 \%$. Meanwhile, ribavirin showed a benefit in decreasing the mortality rate of SARS patients. However, there were some limitations to these studies, namely, the majority of the included studies were cohort studies, with the exception of one RCT; and the treatment dosage, frequency, and administration routes were variable (22). Latterly, Morra et al. revealed that monitoring adverse effects carefully, especially anaemia, bradycardia, diarrhoea, and transaminitis, the rapid initiation of ribavirin and interferon combination may have effects in patients with MERS (32). However, this study only included case reports, case series, and observational studies, and was limited by the small sample size of the included studies (32). Recently, Zhang et al. conducted a systematic review of all therapeutic options associated with coronavirus infections. They suggest a variety of nutrients, antiviral agents, ribonucleic acid virus vaccines, and convalescent plasma based on SARS/MERS experiences, SARS-CoV-2 in-vitro results, or COVID-19 cases. However, they came to no conclusion with regard to a therapy that could effectively treat COVID-19 (20).

In the described systematic review, we attempt to perform a comprehensive manual search of WHO and CDC news, pre-printing websites, and clinical trial registers, in addition to searching electronic databases, to ensure the involvement of all relevant studies. We acknowledge that heterogeneity between study types, conditions, interventions, comparisons, and outcomes is inevitable, and will make performing an appropriate meta-analysis challenging. We will overcome this issue through subgroup analyses of distinct situations, such as indications, interventions, and study designs. Furthermore, the probable absence of large RCTs evaluating treatments for COVID-19 will be another limitation. We conducted a preliminary search that found that only a limited number of RCTs reported the efficacy and safety of antiviral agents for patients with confirmed COVID-19, and three RCTs documented inconsistent results for hydroxychloroquine (33-35). Chen et al. documented that hydroxychloroquine therapy showed a radiographical benefit (34). Tang et al. and Chen $e t a l$. revealed that hydroxychloroquine did not benefit SARS-CoV-2 eradication (33,35), and caused more adverse events, especially diarrhoea (33). Furthermore, two RCTs showed that lopinavir/ritonavir monotherapy did not significantly reduce the viral load of SARS-CoV-2 or improve clinical outcomes over supportive care for hospitalised adult patients with mild/moderate (36) or severe COVID-19 (37). Recently, three RCTs documented that remdesivir was associated with shorter time to recovery $(38,39)$ as opposed to clinical benefits (39) in hospitalised adult patients with COVID-19. No significant difference was noted between the short course ( 5 days) and long course (10 days) of remdesivir (40). In addition, we will perform a quality analysis of the retrieved evidence to evaluate the reliability of recommendations.

In summary, we will perform a systematic review to collect comprehensive evidence of currently available therapeutic options in SARS-CoV, MERS-CoV, and SARS-CoV-2-infected patients. The meta-analysis will evaluate the efficacy and safety of these antiviral agents, such as remdesivir, chloroquine and hydroxychloroquine, lopinavir/ritonavir, ribavirin, arbidol, and interferons, based on retrieved studies. Our study will allow firm recommendations to be drawn with regard to the best choice of treatment for COVID-19.

\section{Acknowledgments}

Funding: This study was funded by the Pharmacy Administration and Rational Drug Use of COVID-19 Treatments at Shanghai Jiao Tong University School of Medicine (JDYX2020KYZX009), WU JIEPING Medical Foundation (320.6750.2020-04-31), Program for Key but Weak Disciplines of Shanghai Municipal Commission of Health and Family Planning (2016ZB0304), Cultivation Fund of Clinical Research of Renji Hospital (PY2018III-06), Research Funds of Shanghai Health and Family Planning Commission (20184Y0022, 20194Y0007), 
and Clinical Pharmacy Innovation Research Institute of Shanghai Jiao Tong University School of Medicine (CXYJY2019ZD001, CXYJY2019QN004).

\section{Footnote}

Reporting Checklist: The authors have completed the PRISMA-P reporting checklist. Available at http://dx.doi. org/10.21037/atm-20-2340

Peer Review File: Available at http://dx.doi.org/10.21037/ atm-20-2340

Conflicts of Interest: All authors have completed the ICMJE uniform disclosure form (available at http://dx.doi. org/10.21037/atm-20-2340). ZCG and YTY serve as unpaid section editors of Annals of Translational Medicine from Oct 2019 to Sep 2020. The other authors have no conflicts of interest to declare.

Ethical Statement: The authors are accountable for all aspects of the work with regard to ensuring that questions related to the accuracy or integrity of any part of the work are appropriately investigated and resolved. Ethical approval is not required because the manuscript is a protocol of a systematic review and meta-analysis.

Open Access Statement: This is an Open Access article distributed in accordance with the Creative Commons Attribution-NonCommercial-NoDerivs 4.0 International License (CC BY-NC-ND 4.0), which permits the noncommercial replication and distribution of the article with the strict proviso that no changes or edits are made and the original work is properly cited (including links to both the formal publication through the relevant DOI and the license). See: https://creativecommons.org/licenses/by-nc-nd/4.0/.

\section{References}

1. Zhou P, Yang XL, Wang XG, et al. A pneumonia outbreak associated with a new coronavirus of probable bat origin. Nature 2020;579:270-3.

2. Chen Y, Liu Q, Guo D. Emerging coronaviruses: Genome structure, replication, and pathogenesis. J Med Virol 2020;92:418-23.

3. Coronavirus disease 2019 (COVID-19) Situation Report-45. [web page]. World Health Organization. Available online: https://www.who.int/docs/default-source/ coronaviruse/situation-reports/20200305-sitrep-45covid-19.pdf?sfvrsn=ed2ba78b_2

4. Chan JF, Kok KH, Zhu Z, et al. Genomic characterization of the 2019 novel human-pathogenic coronavirus isolated from a patient with atypical pneumonia after visiting Wuhan. Emerg Microbes Infect 2020;9:221-36.

5. Zhu Z, Chu H, To KK, et al. Clinical features of patients infected with 2019 novel coronavirus in Wuhan, China. Emerg Microbes Infect 2020;395:497-506.

6. Wang D, Hu B, Hu C, et al. Clinical Characteristics of 138 Hospitalized Patients With 2019 Novel CoronavirusInfected Pneumonia in Wuhan, China. JAMA 2020;323:1061-9.

7. Mahase E. Coronavirus covid-19 has killed more people than SARS and MERS combined, despite lower case fatality rate. BMJ 2020;368:m641.

8. Lu H. Drug treatment options for the 2019-new coronavirus (2019-nCoV). Biosci Trends 2020;14:69-71.

9. Leong HN, Ang B, Earnest A, et al. Investigational use of ribavirin in the treatment of severe acute respiratory syndrome, Singapore, 2003. Trop Med Int Health 2004;9:923-7.

10. Chen Q, Zhang LL, Yu DX, et al. A field trial for evaluating the safety of recombinant human interferon alpha-2b for nasal spray. Zhonghua Shi Yan He Lin Chuang Bing Du Xue Za Zhi 2005;19:211-5.

11. Loutfy MR, Blatt LM, Siminovitch KA, et al. Interferon alfacon-1 plus corticosteroids in severe acute respiratory syndrome: a preliminary study. JAMA 2003;290:3222-8.

12. Chu CM, Cheng VC, Hung IF, et al. Role of lopinavir/ ritonavir in the treatment of SARS: initial virological and clinical findings. Thorax 2004;59:252-6.

13. Chan KS, Lai ST, Chu CM, et al. Treatment of severe acute respiratory syndrome with lopinavir/ritonavir: a multicentre retrospective matched cohort study. Hong Kong Med J 2003;9:399-406.

14. Momattin H, Al-Ali AY, Al-Tawfiq JA. A Systematic Review of therapeutic agents for the treatment of the Middle East Respiratory Syndrome Coronavirus (MERSCoV). Travel Med Infect Dis 2019;30:9-18.

15. Arabi YM, Shalhoub S, Mandourah Y, et al. Ribavirin and Interferon Therapy for Critically Ill Patients with Middle East Respiratory Syndrome: A Multicenter Observational Study. Clin Infect Dis 2020;70:1837-44.

16. Vincent MJ, Bergeron E, Benjannet S, et al. Chloroquine is a potent inhibitor of SARS coronavirus infection and spread. Virol J 2005;2:69.

17. Gao J, Tian Z, Yang X. Breakthrough: Chloroquine 
phosphate has shown apparent efficacy in treatment of COVID-19 associated pneumonia in clinical studies. Science 2020;14:72-3.

18. Sheahan TP, Sims AC, Graham RL, et al. Broad-spectrum antiviral GS-5734 inhibits both epidemic and zoonotic coronaviruses. Sci Transl Med 2017;9:eaal3653.

19. Khamitov RA, Loginova S, Shchukina VN, et al. Antiviral activity of arbidol and its derivatives against the pathogen of severe acute respiratory syndrome in the cell cultures. Vopr Virusol 2008;53:9-13.

20. Zhang L, Liu Y. Potential interventions for novel coronavirus in China: A systematic review. J Med Virol 2020;92:479-90.

21. Morse JS, Lalonde T, Xu S, et al. Learning from the Past: Possible Urgent Prevention and Treatment Options for Severe Acute Respiratory Infections Caused by 2019nCoV. Chembiochem 2020;21:730-8.

22. Momattin H, Mohammed K, Zumla A, et al. Therapeutic options for Middle East respiratory syndrome coronavirus (MERS-CoV)--possible lessons from a systematic review of SARS-CoV therapy. Int J Infect Dis 2013;17:e792-8.

23. Liberati A, Altman DG, Tetzlaff J, et al. The PRISMA statement for reporting systematic reviews and meta-analyses of studies that evaluate health care interventions: explanation and elaboration. J Clin Epidemiol 2009;62:e1-34.

24. Higgins JP, Altman DG, Gotzsche PC, et al. The Cochrane Collaboration's tool for assessing risk of bias in randomised trials. BMJ 2011;343:d5928.

25. Zhong H, Ni XJ, Cui M, et al. Evaluation of pharmacist care for patients with chronic obstructive pulmonary disease: a systematic review and meta-analysis. Int J Clin Pharm 2014;36:1230-40.

26. Wells GA, Shea B, O'Connell D, et al. The NewcastleOttawa Scale (NOS) for assessing the quality of nonrandomised studies in meta-analyses. [web page]. Ottawa Hospital Research Institute. Available online: http:// www.ohri.ca/programs/clinical_epidemiology/oxford.asp.

27. Zhong H, Zhao XY, Zhang ZL, et al. Evaluation of the efficacy and safety of ceftazidime/avibactam in the treatment of Gram-negative bacterial infections: a systematic review and meta-analysis. Int J Antimicrob Agents 2018;52:443-50.

28. Higgins JP, Green S. Cochrane Handbook for Systematic Reviews of Interventions: Cochrane Book Series. Chichester, UK: John Wiley \& Sons, Ltd., 2008.

29. Higgins JP, Thompson SG, Deeks JJ, et al. Measuring inconsistency in meta-analyses. BMJ 2003;327:557-60.

30. Wang M, Cao R, Zhang L, et al. Remdesivir and chloroquine effectively inhibit the recently emerged novel coronavirus (2019-nCoV) in vitro. Cell Res 2020;30:269-71.

31. Liang C, Tian L, Liu Y, et al. A promising antiviral candidate drug for the COVID-19 pandemic: A minireview of remdesivir. Eur J Med Chem 2020;201:112527.

32. Morra ME, Van Thanh L, Kamel MG, et al. Clinical outcomes of current medical approaches for Middle East respiratory syndrome: A systematic review and metaanalysis. Rev Med Virol 2018;28:e1977.

33. Tang W, Cao Z, Han M, et al. Hydroxychloroquine in patients with mainly mild to moderate coronavirus disease 2019: open label, randomised controlled trial. BMJ 2020;369:m1849.

34. Chen Z, Hu J, Zhang Z, et al. Efficacy of hydroxychloroquine in patients with COVID-19: results of a randomized clinical trial. medRxiv 2020. doi: https://doi. org/10.1101/2020.03.22.20040758.

35. Chen J, Liu D, Liu L, et al. A pilot study of hydroxychloroquine in treatment of patients with moderate COVID-19. Zhejiang Da Xue Xue Bao Yi Xue Ban 2020;49:215-9.

36. Li Y, Xie Z, Lin W, et al. Efficacy and Safety of Lopinavir/ Ritonavir or Arbidol in Adult Patients with Mild/Moderate COVID-19: An Exploratory Randomized Controlled Trial. Med (N Y) 2020. [Epub ahead of print].

37. Cao B, Wang Y, Wen D, et al. A Trial of LopinavirRitonavir in Adults Hospitalized with Severe Covid-19. N Engl J Med 2020;382:1787-99.

38. Beigel JH, Tomashek KM, Dodd LE, et al. Remdesivir for the Treatment of Covid-19 - Final Report. N Engl J Med 2020. doi: 10.1056/NEJMoa2007764.

39. Wang Y, Zhang D, Du G, et al. Remdesivir in adults with severe COVID-19: a randomised, doubleblind, placebo-controlled, multicentre trial. Lancet 2020;395:1569-78.

40. Goldman JD, Lye DCB, Hui DS, et al. Remdesivir for 5 or 10 Days in Patients with Severe Covid-19. N Engl J Med 2020;383:1827-37.

Cite this article as: Zhang ZL, Zhong H, Liu YX, Le KJ, Cui M, Yu YT, Gu ZC, Gao Y, Lin HW. Current therapeutic options for coronavirus disease 2019 (COVID-19)—lessons learned from severe acute respiratory syndrome (SARS) and Middle East Respiratory Syndrome (MERS) therapy: a systematic review protocol. Ann Transl Med 2020;8(22):1527. doi: 10.21037/atm-20-2340 